\title{
Novel Approach to the Diagnosis of Fractures in an Austere Environment Using a Stethoscope and a Cellular Phone
}

\author{
Brett A. Matzek, MD; Phillip T. Fivecoat, DO; Reis B. Ritz, MD \\ From the Department of Emergency Medicine, Carl R. Darnall Army Medical Center, Fort Hood, TX.
}

\begin{abstract}
Background.-Fracture diagnosis in the austere environment where radiographic tests are not available can be a challenge. In the past, a diagnostic technique has been described using a tuning fork and stethoscope to assess decreased sound conduction in the fractured extremity. In this study, we evaluate the use of a cellular phone's vibrate function and a stethoscope to limit equipment carried by expeditionary practitioners.

Objective.-The purpose of this study was to evaluate the accuracy of fracture diagnosis using a cellular phone and stethoscope.

Methods.-This is a pilot study to assess the usefulness of the above technique before clinical implementation. In 3 cadavers, we created fractures of the humerus and femur. Twenty-seven emergency medicine residents and an attending physician performed the diagnostic technique.

Results.-Overall, the use of the cellular phone and stethoscope resulted in a sensitivity of 73\% (95\% confidence interval [CI]: 0.64 to 0.81 ) and a specificity of $83 \%$ (95\% CI: 0.77 to 0.88 ), with a positive predicted value of $68 \%$ (95\% CI: 0.59 to 0.77 ) and a negative predicted value of $86 \%$ (95\% CI: 0.81 to 0.90). Positive likelihood ratio was 4.3 , and negative likelihood ratio was 0.32 .

Conclusions.- The use of a cellular phone and stethoscope may be a useful tool for the diagnosis of fractures in the austere environment. However, further study is needed to validate these findings in the clinical environment.
\end{abstract}

Key words: fracture, auscultation, tuning fork, diagnosis, wilderness medicine, austere medicine, combat medicine

\section{Introduction}

Healthcare providers practicing outside of the hospital setting, such as those participating in expeditionary, sports, or battlefield medicine, are often presented with diagnostic dilemmas. One such dilemma is the accurate diagnosis and disposition of musculoskeletal injuries. These practitioners have a wide range in skill levels and often work in remote locations where they must independently make evacuation and treatment decisions under challenging conditions. They are in constant need of simple, effective, and easily learned diagnostic techniques.

The views expressed in this case study are those of the authors and do not represent the official policy or position of the Department of Defense, the Department of the Army, or the United States Government.

Corresponding author: Brett Matzek, MD, Department of Emergency Medicine, 36000 Darnall Loop, Fort Hood, TX 76544 (e-mail: brett.a. matzek@us.army.mil).
The evaluation of fractures rests mostly on the physical examination. However, beyond the traditional examination, it is sometimes taught that a fracture can be detected using a $128 \mathrm{~Hz}$ tuning fork and a stethoscope. The technique involves placing the vibrating tuning fork distal to the injury and auscultating proximally. Bone with cortical disruption results in a decrease in the sound propagation. Several studies have demonstrated the value of this technique. ${ }^{1-4}$ To minimize the equipment carried by practitioners in an austere environment, we propose changing the vibration source to a more commonly carried tool, a cellular telephone. In this pilot study, we demonstrate a novel approach to the diagnosis of fractures in the humerus and femur using a cellular phone and stethoscope.

\section{Methods}

Using 2 cadavers, fractures were made in 1 humerus and 1 femur each. One additional body was used as a control. 


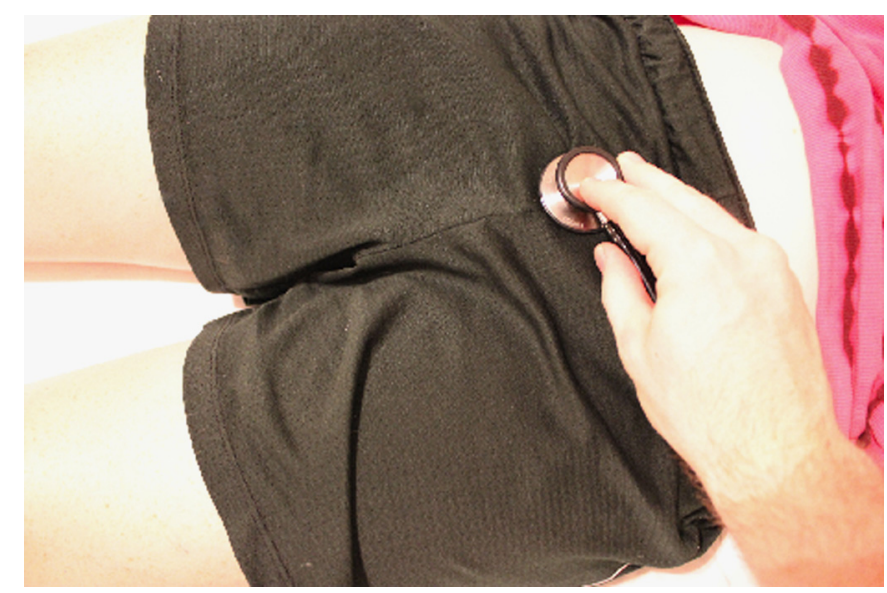

Figure 1. Femur auscultation site at the pubis. (Personal protective equipment excluded for demonstration purposes.)

Fractures were produced with the combined use of a bone saw, hammer, and chisel. A variety of fracture sizes and types were produced including transverse, oblique, and comminuted. Fractures of the humerus were done at the surgical neck whereas the femur fractures were produced just distal to the lesser trochanter. All limbs had the same incision made, to blind the participants. A convenience sample of 27 emergency medicine residents and 1 emergency medicine attending physician performed the diagnostic examination.

The participants had never used the study technique nor had they used the tuning fork technique before their participation in this study. There were no known hearing deficits among the participants. For the femur, participants were asked to auscultate at the pubic symphysis and decide which side was decreased: left, right, or neither (Figure 1). For the humerus, participants were asked to auscultate at the anterior shoulder and decide which side was decreased: left, right, or neither
(Figure 2). The researcher held the vibrating cell phone to the patella for the lower extremities (Figure 3) and to the lateral epicondyle for the upper extremities (Figure 4). The sites of vibration and auscultation resemble previous studies done with the tuning fork technique. Duration of contact was approximately $3 \mathrm{~s}$ per side. The participants were able to request repeats on either side until confident in their examination. To limit amplitude variability, the phone was applied without a case, flush against the bony prominence, with no downward or lateral pressure. The participants were blinded as to which limbs were fractured.

For this study, we used the Apple iPhone 3S (Apple, Cuppertino, CA) and the Littmann Master Classic II Stethoscope (3M Health Care, St. Paul, MN). To make the phone vibrate repeatedly, the iVibe application was used (Robot Mouse, West Chester, PA). Measures of diagnostic accuracy were calculated using GraphPad Prism version 5.04 for Windows (GraphPad Software,

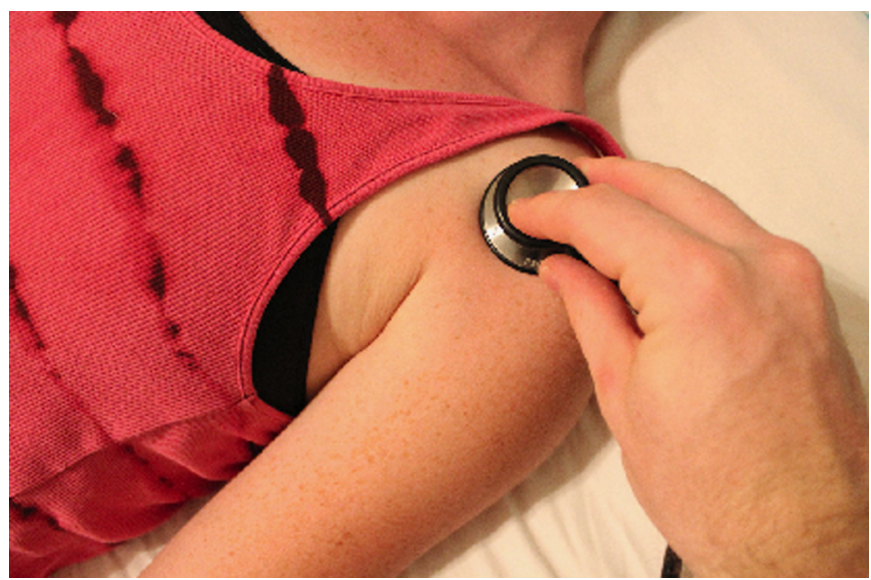

Figure 2. Humerus auscultation site at the anterior shoulder. (Personal protective equipment excluded for demonstration purposes.) 


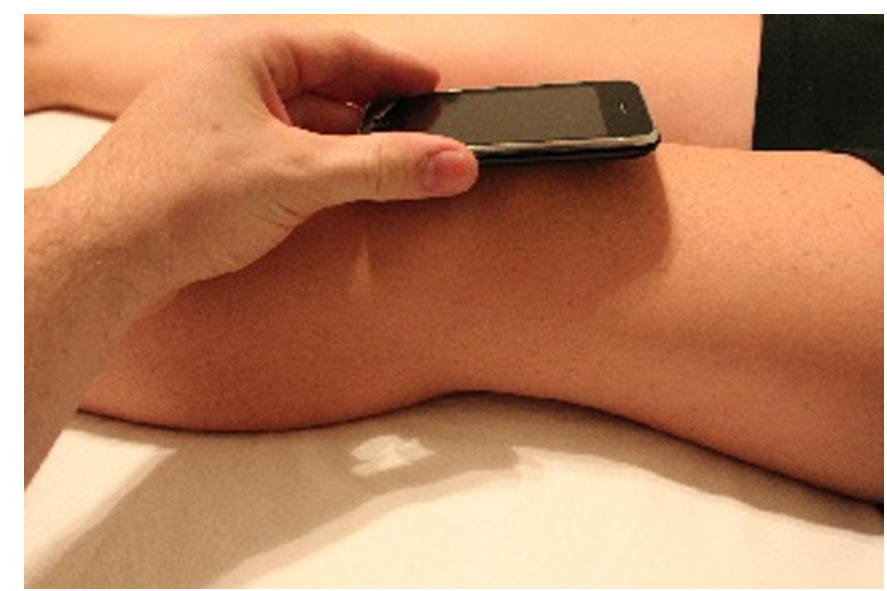

Figure 3. Femur vibration site at the patella. (Personal protective equipment excluded for demonstration purposes.)

San Diego, CA; www.graphpad.com). This study was exempt from the Institutional Review Board at the University of Texas Health Science Center at Houston.

\section{Results}

The study included 3 cadaver models and 28 participants, including 27 emergency medicine residents of all year groups and 1 emergency medicine attending physician. Among the 3 cadaver models there were 2 fractured humeri and 2 fractured femurs. Each participant examined 12 extremities, resulting in 336 total examinations (Table 1). Overall, the use of the cellular phone and stethoscope resulted in a sensitivity of $73 \%$ (95\% confidence interval [CI]: 0.64 to 0.81 ) and a specificity of $83 \%$ (95\% CI: 0.77 to 0.88 ), with a positive predictive value of $68 \%$ (95\% CI: 0.59 to 0.77 ), a negative predictive value of $86 \%$ (95\% CI: 0.81 to 0.90 ), a positive likelihood ratio of 4.3 , and a negative likelihood ratio of 0.32 (Table 2).

\section{Discussion}

The use of tuning forks to auscultate fractures has been studied several times in the past ${ }^{1-4}$ and found to be moderately sensitive and specific. Bache and Cross ${ }^{1}$ used a stethoscope and $128 \mathrm{~Hz}$ tuning fork to diagnose fractures of the femoral neck and shaft and found an accuracy of $87 \%$ for femoral neck fractures. Misurya et $\mathrm{al}^{3}$ used a 128 $\mathrm{Hz}$ tuning fork and a pediatric stethoscope to diagnose fractures to the tibia and to the femoral neck and shaft. They were able to detect fractures in $94 \%$ of patients. Moore ${ }^{4}$ used the same equipment on a wide array of bones from the femur to the clavicle and found an overall sensitivity of $83 \%$, specificity of $80 \%$, positive LR of 4.2, and a negative LR of 0.2 for the diagnosis of fractures. Jawad et $\mathrm{al}^{2}$ added an objective measurement by using a tuning fork and an electronic stethoscope to detect changes in amplitude, and found an accuracy of $80 \%$. Among these studies, the reasons for inaccurate results included bilateral pubic rami fractures, chronic osteomyelitis, obesity,

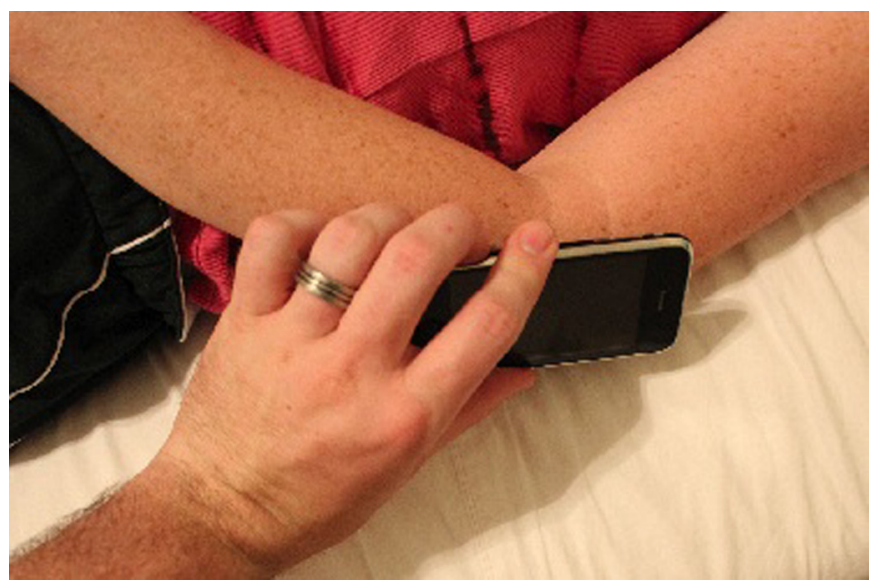

Figure 4. Humerus vibration site at the lateral epicondyle. (Personal protective equipment excluded for demonstration purposes.) 
Table 1. Summary of examination findings

\begin{tabular}{lccc}
\hline & Fracture & No fracture & Total \\
\hline Positive test & 82 & 38 & 120 \\
Negative test & 30 & 186 & 216 \\
Total & 112 & 224 & 336 \\
\hline
\end{tabular}

posterior hip fracture, fracture impaction, avulsion, and buckle fractures. ${ }^{1-4}$ Our study found similar results with an overall sensitivity of $73 \%$ and specificity of $83 \%$ and a negative predictive value of $86 \%$.

\section{STUDY LIMITATIONS}

This is a test-of-concept study to evaluate the use of the cellular phone as a vibratory source to diagnose fractures by auscultation. This study was limited to 3 cadaver models and a convenience sample of emergency medicine residents and staff. It was blinded only to the participants. To minimize bias, the researchers should have been blinded; however, the production of the fractures made this difficult. The fractures were simulated and may not exactly resemble naturally occurring fractures. In addition, the cadavers may not conduct sound the same way a living specimen would owing to tissue changes postmortem. The cadavers were on large metal tables, which are suspected to have caused an increase in sound conduction. Towels were placed beneath the upper extremities and pelvis to minimize this variable.

Table 2. Statistical analysis

\begin{tabular}{lc}
\hline Statistical analysis & Value $(95 \%$ confidence interval) \\
\hline Sensitivity & $0.73(0.64-0.81)$ \\
Specificity & $0.83(0.77-0.88)$ \\
Positive predictive value & $0.68(0.59-0.77)$ \\
Negative predictive value & $0.86(0.80-0.90)$ \\
Positive likelihood ratio & 4.31 \\
Negative likelihood ratio & 0.32 \\
\hline
\end{tabular}

\section{Conclusions}

Although the tuning fork and stethoscope technique is taught to physicians participating in expeditionary medicine, few use it in practice. Medicine in the austere environment focuses on limiting equipment and using equipment with multiple uses. In this case, the tuning fork has limited utility. However, most expedition physicians will carry a stethoscope and a cell phone. The ability to diagnose fractures will aid in disposition and evacuation decisions. With a negative predictive value of $86 \%$, if the physician does not hear a difference between the injured and uninjured extremity, evacuation could possibly be delayed. That is particularly important in the deployed environment where evacuation is both limited and dangerous. This method is a simple, cost-effective adjunct to the clinical examination.

In this study, we found an overall sensitivity of $73 \%$ and specificity of $83 \%$. The next step is to utilize this method in the emergency department or orthopedic clinic to assess its accuracy for living tissue with a variety of fracture types and locations.

\section{Acknowledgments}

We thank Sarah Matzek for her assistance in manuscript preparation and editing, and Peter Moffett for his assistance with statistical analysis.

\section{References}

1. Bache JB, Cross AB. The Barford test: useful diagnostic sign in fractures of the femoral neck. Practitioner. 1984;228:305-308.

2. Jawad Z, Odumala A, Jones M. Objective sound wave amplitude measurement generated by a tuning fork. An analysis of its use as a diagnostic tool in suspected femoral neck fractures. Injury. 2012;43:835-837.

3. Misurya RK, Khare A, Mallick A, Sural A, Vishwakarma GK. Use of tuning fork in diagnostic auscultation of fractures. Injury. 1987;18:63-64.

4. Moore MB. The use of a tuning fork and stethoscope to identify fractures. J Athletic Training. 2009;44:272-274. 